Warrington and Warrington. HCA Healthcare Journal of Medicine (2020) 1:4

https://doi.org/10.36518/2689-0216.1083

\title{
Education
}

\section{An Inexpensive Adult Lumbar Puncture Task- Trainer with Simulated Cerebrospinal Fluid}

Steven Warrington, MD, ${ }^{1}$ Anne Marie Warrington

\section{Abstract}

\section{Background}

Simulation is beneficial in achieving competence and success in the performance of procedures. Unfortunately, costs associated with purchasing simulation equipment is a significant barrier to implement this training. The objective was to create a model for lumbar puncture that allowed performance of the procedure using anatomic landmarks, ability to obtain cerebrospinal fluid, and measure opening pressure.

\section{Methods}

A model was built using several readily available and low-cost items and was iterated based on feedback. The task trainer was used as part of multiple educational sessions and simulations with different groups of learners, including residents and medical students.

\section{Results}

A reusable task trainer was built to simulate the performance of a lumbar puncture that met our objective of using anatomic landmarks with the ability to collect simulated cerebrospinal fluid and measure opening pressure. The model had a low fixed cost for the initial investment (less than \$75) and minimal variable costs.

\section{Conclusions}

An innovative and easy to make low-cost model is presented as an option to use for lumbar puncture training at institutions with limited resources. This do-it-yourself model is unique in providing learners the opportunity to obtain cerebrospinal fluid and measure opening pressure. The model is easy to transport and can be implemented as part of educational sessions and simulation scenarios.

\section{Keywords}

simulation training; education, medical, graduate; internship and residency; spinal puncture/ methods; diagnostic techniques and procedures; task performance and anaylsis; task trainer: models, educational; low-cost; do-it-yourself

\section{Introduction}

Task-trainers have been shown to be beneficial in achieving competence in the performance of lumbar puncture (LP).' Despite the proven benefits, the costs associated with purchasing such equipment pose a significant barrier to implementing this training, which has been commented on before. ${ }^{2} \mathrm{~A}$ brief electronic search yielded multiple commercial products readily available, ranging from $\$ 1,195-3,999.3-8$

(Table 1) There have been various approaches to combat the monetary barriers present with implementing simulation-based training such as building low-cost do-it-yourself models. Examples of procedures with documented innovative low-cost models include perimortem cesarean section and sinus surgery. ${ }^{9-11}$

Multiple authors have previously published on low-cost models for the performance of lumbosacral spine procedures. ${ }^{2,12}$ Existing models are not ideal for the performance of a LP based on anatomic landmarks, nor do they allow the ability to collect simulated cerebrospinal fluid (CSF) or measure opening pressure. Despite multiple educational resources existing for LP

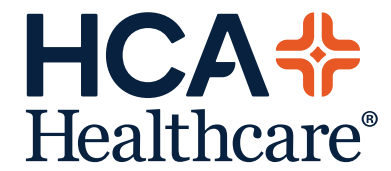

\section{www.hcahealthcarejournal.com}

(c) 2020 HCA Physician Services, Inc. d/b/a Emerald Medical Education
HCA Healthcare Journal of Medicine 
Table 1. Commercially available products for lumbar puncture training with prices listed (not including shipping and handling).

\begin{tabular}{llc}
$\begin{array}{l}\text { Commercially } \\
\text { available }\end{array}$ & Product name & Cost \\
\hline Gaumard & Lumbar Puncture Skills Trainer S411 & $\$ 1,195.00$ \\
\hline Limbs \& Things & $\begin{array}{l}\text { Pediatric Lumbar Puncture Simulator } \\
\text { (Product No. KKM43D) }\end{array}$ & $\$ 1,600.00$ \\
\hline Limbs \& Things & $\begin{array}{l}\text { Lumbar Puncture Model } \\
\text { (Product No. 61000) }\end{array}$ & $\$ 1,900.00$ \\
\hline Simulab & Lumbar Puncture Trainer & $\$ 1,975.00$ \\
\hline GT Simulators & KKAmerica Lumbar Puncture Simulator II & $\$ 2,107.00$
\end{tabular}

\begin{tabular}{lll}
\hline Blue Phantom/CAE Lumbar Puncture and Spinal Epidural $\$ 3,999.00$ \\
\hline
\end{tabular}

training, none overcame the barriers of cost while providing a model with the desired features. ${ }^{13,14}$

This article presents a novel and inexpensive model for LP training that allows for the simulated performance of the procedure based on anatomic landmarks with the potential to measure opening pressure and collect simulated CSF.

\section{Methods}

Construction of the model requires the following supplies and equipment: 1) One plastic tote with a base large enough to serve as an outline for an adult back (authors' tote had a base of approximately 14 in $\times 25$ in) with height of at least 6 inches; 2 ) One queen sized memory foam pillow (authors' pillow was 16 in $\times 29$ in with a 5 inch loft) with a pillow case containing a zipper to open/close the case that goes around the foam; 3) A plastic skeleton spine or other material to simulate a spine (authors' Halloween skeleton was 12 inches in length and 1.5 inch circumference); 4) Heavy duty scissors, tape and marker; and 5) kitchen sealable plastic bags in quart and gallon size (authors' bags were 6 in $\times 9$ in and 12 in $\times 10$ in). Ideally, the foam pillow should have a case that can mimic skin to some degree. However if one is unable to be found, then material to make simulated skin will be required. One option is to use a commercially available product due to its low cost and availability (Smooth-on Dragon Skin $30^{\mathrm{TM}}$ ).

If a silicone skin is desired, then the first step will be to follow the instructions of a commercially available product to develop a silicone skin that is larger than one face of the pillow. The next step is to modify the base of the tote into a shape that will provide some structure and anatomic landmarks of the iliac crests. Cut an outline of an "I" (that has a thick top and bottom) on the base of the tote while in a vertical position. The top of the "I" serves as the shoulders/scapulae while the bottom of the "I" serves as the iliac crests and sacrum. Also, cut 2-3 small (approximately 0.5 in $\times 1.25$ in) midline rectangles that will serve as the spaces for the needle to pass through between the spinous processes. Those rectangles should align at the appropriate clinical level with where the tote's simulated iliac crests are located. Excess material can be removed, and the base structure should be done.

Next, modify the foam of the pillow to serve as the soft tissue that overlies the structure and landmarks, which will also provide some resistance for the spinal needle. One face of the foam will remain intact. Figure 1 demonstrates what the open side of the foam pillow will look like. An initial longitudinal cut should be made along the midline at approximately $80 \%$ of the depth of the pillow. Then extend the base of 


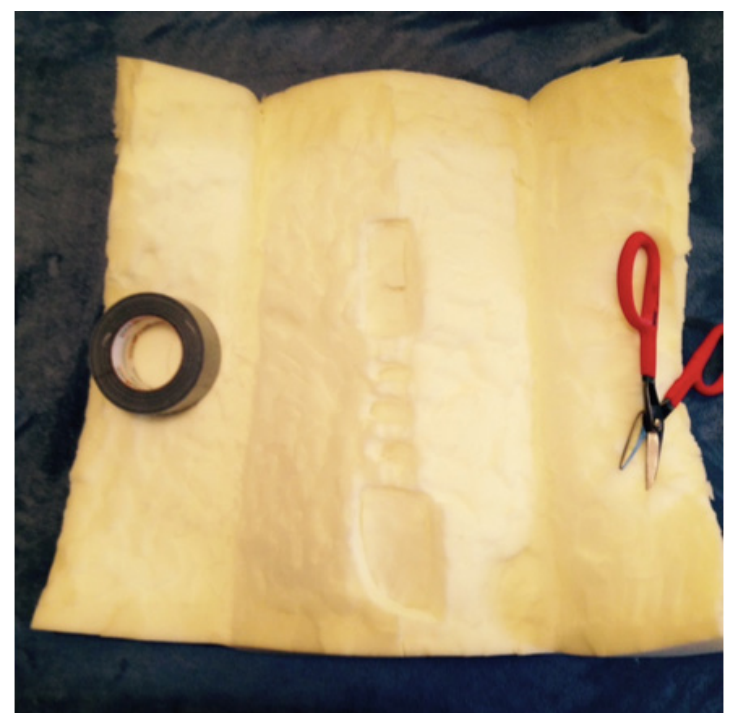

Figure 1. After cutting the pillow lengthwise, make cuts so it folds open. Cut portions out of the midline that will allow the simulated bones to sit in.

the cut towards each side of the pillow leaving approximately $1 / 2$ inch of foam intact. Cut out spaces in the foam on the now open surface for the simulated vertebrae and sacrum to sit in. Embed the materials that will simulate the vertebrae and sacrum (Figure 2), which will allow a more realistic sensation on palpation by decreasing depth to spinous process. It also ensures that foam will remain between vertebrae to give resistance and feedback while advancing the spinal needle. Many materials or plastics may suffice. The authors used a Halloween skeleton's vertebrae and foot (for the sacrum), which provided appropriate haptic sensation. It is important to make sure the areas between the vertebrae correlate with areas cut along the midline of the plastic tote in order to allow the passage of the spinal needle.

Figure 3 shows the foam with the inset simulated spine and sacrum wrapped around the outside of the plastic tote that simulates the soft tissue overlying the lateral edge of the iliac crests, shoulders, and hips during palpation. The lateral border of the pillow also serves to provide structure for flanks of the task-trainer in the space that would otherwise be empty.

The structure and outline of the model is now complete. When it is time to use the model, the plastic re-sealable bags will be used to simulate the feel of passing through ligamentum flavum and to contain water that serves as simulated

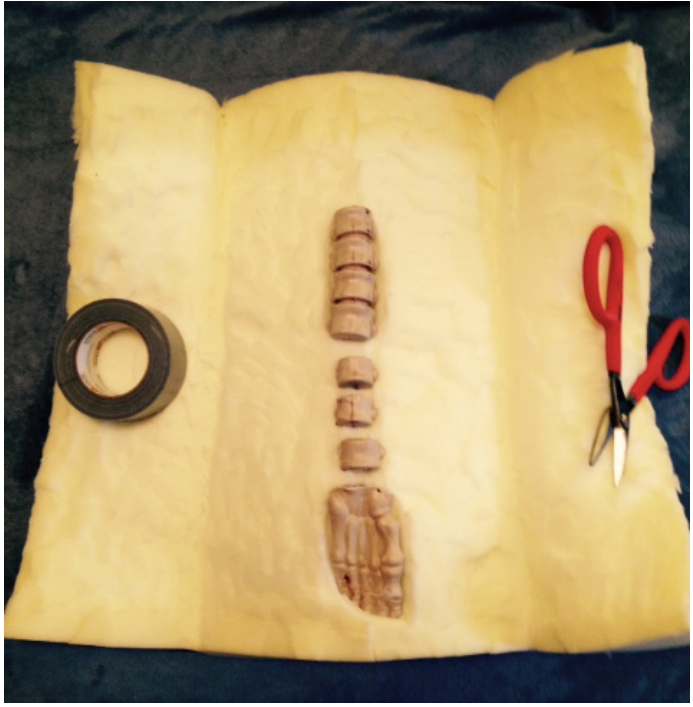

Figure 2. Material to simulate vertebrae and sacrum are embedded in foam. 


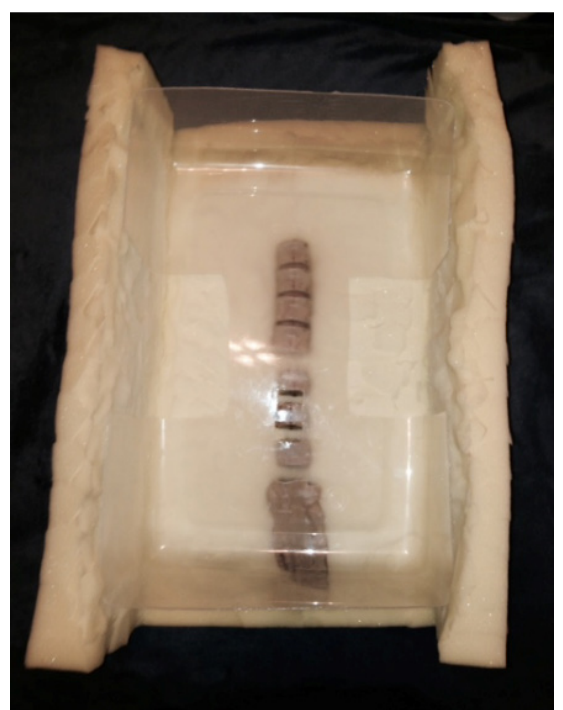

Figure 3. The foam wraps around the tote providing structure and "soft tissue" for palpation.

CSF. The authors used a quart sized resealable plastic bag filled with water contained within a gallon resealable plastic bag. The purpose of using two bags is that following puncture the fluid from the inside bag is effectively prevented from leaking onto the foam or within the task trainer by the second bag. The one gallon re-sealable bag is then secured with tape to the inside plastic surface overlying the openings made for passage of the spinal needle, while the smaller resealable bag is filled with water, closed, and set inside the larger bag. (Figure 4)

\section{Results}

A novel model for simulated performance of
LP through the use of anatomic landmarks was generated for under $\$ 75$. The model met the objectives to have the procedure performed based on anatomic landmarks (Figure 5) and allowed the measurement of opening pressure and collection of CSF. Variable costs for ongoing use of the initial model are related to replacing the resealable bags and spinal needles and would be estimated to be less than $\$ 0.50$ per user. Construction of the model took less than an hour once all materials were collected.

Following the construction of the model, it was implemented as a task-trainer in educational sessions on LP and as an adjunct in simulation

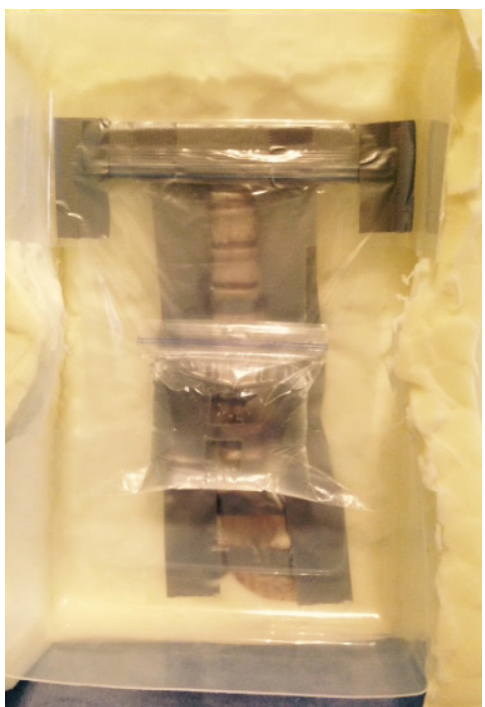

Figure 4. A larger sealable bag is taped to the inside of the tote, which contains a smaller sealable bag filled with water. 


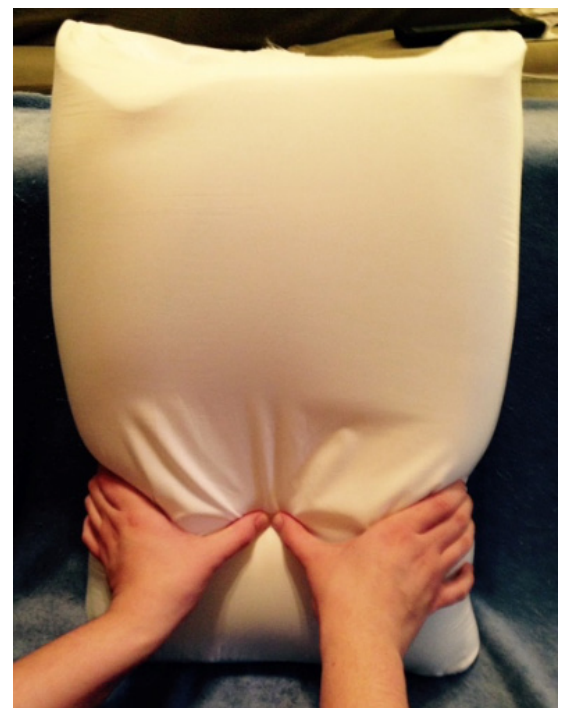

Figure 5. The finished task-trainer allows for palpation of anatomic landmarks for procedural performance.

scenarios. Anecdotally, learners found the model to be useful and of benefit to their skills and comfort related to performing LP, which was identified during debriefing.

Limitations with this model include decreased fidelity as compared to certain commercial models. Additionally, it comes with a time commitment related to construction that would not be seen with a commercial model. However, this model overcomes the monetary barrier, potentially preventing implementation of simulation-based LP training on a model that provides anatomic landmarks, has the capability of CSF return, and opening pressure measurements.

\section{Discussion}

The purpose of the paper was to describe the construction of a LP model that would meet the following specific objectives: 1) Allow performance of a simulated LP based on anatomic landmarks; 2) Allow measurement of opening pressure; and 3) Allow the collection of simulated CSF. The model was relatively inexpensive and easy to build while meeting the objectives. Objective 1 was met by having palpable anatomic landmarks such as the iliac crests. Objective 2 was met by having simulated cerebrospinal fluid to collect in the manometer with an obtainable measurement. Objective 3 was met by using tap water that could be collected and kept from entering the simulated target anatomic location. Despite some fidelity being sacrificed, a fixed cost of less than $\$ 75$ may allow for cost-related barriers to be overcome.

From the authors' experience, a second model was built in the same fashion in order to support educational sessions with more than 3-4 learners. A second station was simultaneously run and learners did not waste time waiting to use the model. A second facilitator was required to supervise and assist with the procedure at the second station. An additional lesson learned was to utilize air when simulating injecting lidocaine and dried betadine swabs for the site preparation, so the foam of the task-trainer did not get wet or ruined.

\section{Conclusion}

The authors believe this contribution is significant to existing literature and resources used at many institutions that do not have funds to purchase LP models but still desire the ability to simulate the procedure on an adult using anatomic landmarks and resulting in the collection of simulated cerebrospinal fluid. In choosing to build a model, the learner can expect to find it to be a somewhat easy task while needing to make sure it is anatomically placed properly.

\section{Conflicts of Interest}

The authors declare they have no conflicts of interest. 
Dr. Warrington is an employee of Orange Park Medical Center, a hospital affiliated with the journal's publisher.

This research was supported (in whole or in part) by HCA Healthcare and/or an HCA Healthcare affiliated entity. The views expressed in this publication represent those of the author(s) and do not necessarily represent the official views of HCA Healthcare or any of its affiliated entities.

\section{Author Affiliation}

1. Orange Park Medical Center, Orange Park, $\mathrm{FL}$

\section{References}

1. Conroy SM, Bond WF, Pheasant KS, Ceccacci N. Competence and retention in performance of the lumbar puncture procedure in a task trainer model. Simul Healthc. 2010 Jun;5(3):133-138. https://doi.org/10.1097/SIH.0b013e3181dc040a

2. Faulkner AR, Bourgeois AC, Bradley YC, Pasciak AS. A robust and inexpensive phantom for fluoroscopically guided lumbar puncture training. Simul Healthc. 2015 Feb;10(1):54-58. https://doi. org/10.1097/SIH.0000000000000066

3. Blue Phantom Lumbar Puncture And Spinal Epidural Training Model. BluePhantom. https:// www.bluephantom.com/product/Lumbar-Puncture-and-Spinal-Epidural.aspx. Accessed June 26, 2020.

4. Lumbar Puncture Simulator II. GTsimulators. com. https://www.gtsimulators.com/lumbar-puncture-simulator-ii-p/kk-m43b.htm?gclid=EAlalQobChMludfdjuD36QIV4suGChOy7AL-EAQYASABEgLfKfD BwE. Accessed June 26, 2020.

5. S411 Lumbar Puncture Trainer. Gaumard Scientific. https://www.gaumard.com/s411. Accessed June 26, 2020.

6. Lumbar Puncture Model. Limbs \& Things. https://limbsandthings.com/us/products/61000/61000-lumbar-puncture-model. Accessed June 26, 2020.

7. Pediatric Lumbar Puncture Simulator. Limbs \& Things. https://limbsandthings.com/us/products/kkm43d/kkm43d-pediatric-lumbar-puncture-simulator. Accessed June 26, 2020.

8. Lumbar Puncture Trainer. Simulab. https://www. simulab.com/products/lumbar-puncture-trainer. Accessed June 26, 2020.

9. Sampson CS, Renz NR, Wagner JC. An inexpensive and novel model for perimortem cesarean section. Simul Healthc. 2013 Feb;8(1):49-51. https://doi.org/10.1097/SIH.0b013e318271489c
10. Kassab E, Tun JK, Arora S, et al. "Blowing up the barriers" in surgical training: exploring and validating the concept of distributed simulation. Ann Surg. 2011 Dec;254(6):1059-1065. https://doi. org/10.1097/SLA.Ob013e318228944a

11. Malekzadeh S, Pfisterer MJ, Wilson B, Na $\mathrm{H}$, Steehler MK. A novel low-cost sinus surgery task trainer. Otolaryngol Head Neck Surg. 2011 Oct;145(4):530-533. https://doi. org/10.1177/0194599811413373

12. Bellingham GA, Peng PW. A low-cost ultrasound phantom of the lumbosacral spine. Reg Anesth Pain Med. 2010;35(3):290-293. https://doi. org/10.1097/AAP.0b013e3181c75a76

13. Wathen P, Johnson M, O'Rorke J, Lawrence V. Lumbar puncture procedure module. MedEdPORTAL. 2011;7:8201. https://doi.org/10.15766/ mep 2374-8265.8201

14. Smyth P, Jeerakathil T, Roberts T. Lumbar puncture teaching skills objective structured clinical examination (OSCE) station. MedEdPORTAL. 2014;10:9800. https://doi.org/10.15766/ mep 2374-8265.9800 